12

\title{
Моделирование высокочастотной фотопушки для генерации ультракоротких пучков
}

\author{
() Д.А. Никифроров, ${ }^{1}$ А.Е. Левичев, ${ }^{1,2}$ А.М. Барняков, ${ }^{1}$ А.В. Андрианов, ${ }^{1,2}$ С.Л. Самойлов ${ }^{1}$ \\ ${ }^{1}$ Институт ядерной фризики им. Г.И. Будкера СО РАН, \\ 630090 Новосибирск, Россия \\ ${ }^{2}$ Новосибирский национальный исследовательский государственный университет, \\ 630090 Новосибирск, Россия \\ e-mail: nikdanila@bk.ru
}

(Поступило в Редакцию 7 июня 2016 г. В окончательной редакции 24 сентября 2017 г.)

Выполнен расчет высокочастотной фотопушки для генерации ультракоротких пучков электронов с целью использования в экспериментах по быстрой электронной дифрактоскопии, кильватерному ускорению и разработке ускоряющих структур миллиметрового диапазона длин волн. Приведено обоснование необходимых параметров пучка на выходе фотопушки для каждого из экспериментов. Показан общий вид фотопушки, рассчитаны ее электродинамические характеристики, получено распределения ускоряющего поля. Исходя из необходимых конечных параметров пучка произведен анализ динамики частиц. Подобраны оптимальные начальные характеристики пучка и амплитуды полей. Сделано заключение о достигнутых параметрах пучка.

DOI: 10.21883/JTF.2018.04.45731.1914

\section{Введение}

В настоящее время во многих научных лабораториях мира разрабатываются универсальные ускорительные комплексы на основе источников ультракоротких пучков для проведения различных исследований как в области ускорительной техники, так и в других областях науки [1-3]. Основой источника ультракоротких пучков, как правило, является высокочастотная (ВЧ) фотопушка. Принцип ее действия заключается в получении пучка электронов из фотокатода под действием лазерного излучения с последующим ускорением в электромагнитном поле резонаторов. Такой метод генерации пучков обладает рядом особенностей.

1. Длина электронного пучка определяется длительностью лазерного импульса. Развитие современных лазеров позволяет получать импульсы с длительностью в несколько десятков фемтосекунд [4].

2. Начальный размер пятна лазера на фотокатоде влияет на эмиттанс пучка, поскольку фактически оно определяет его начальное значение.

3. Влияние объемного заряда можно существенно минимизировать путем увеличения градиента ускорения в самой пушке, который зависит не только от входной ВЧ мощности, но и от начальной фазы ускорения [5].

4. Оптимальный ввод ВЧ мощности позволяет уменьшить влияние поперечных сил электромагнитного поля, действующих на заряд вследствие нарушения аксиальной симметрии системы [5].

5. Заряд пучка электронов зависит от типа фотокатода, a также от параметров лазера (энергия в импульсе, длина волны). Варьирование параметров лазера и выбор фотокатода дает возможность работы в большом диапазоне зарядов сгенерированного пучка (от $100 \mathrm{fC}$ до нескольких $\mathrm{nC}$ ) [6,7].
Настоящая работа посвящена расчету высокочастотной фотопушки $S$-диапазона в Институте ядерной физики им. Г.И. Будкера СО РАН (ИЯФ СО РАН). На ее основе планируется проведение исследований по получению пучков для различных приложений, среди которых основными являются: исследования быстропротекающих процессов на основе электронной дифрактоскопии, исследования в области плазменного кильватерного ускорения, генерация пучков электронов для возбуждения электромагнитных полей миллиметровых длин волн. Параметры пучка для данных задач сильно отличаются. При этом заряд пучка и его начальная длительность в основном зависят от свойств фотокатода, энергии и длительности импульса лазера. Однако такие характеристики, как конечная длительность пучка, эмиттанс, энергия, угловое расхождение, поперечный размер и т. П., являются результатом в первую очередь динамики частиц и свойств ВЧ фотопушки. Ниже приводятся расчеты необходимых параметров пучка для каждого из приложений, описание разрабатываемой ВЧ фотопушки и расчеты динамики пучка без рассмотрения свойств фотокатода и лазерной системы. Несмотря на то что на данный момент существуют лазеры с длительностью импульса в десятки фемтосекунд [4], начальная длина пучка выбиралась в районе нескольких пикосекунд, что позволит использовать более доступный и недорогой лазер.

\section{Расчет параметров пучка ВЧ фотопушки}

С помощью метода быстрой электронной дифрактоскопии можно изучать процессы в образце с временным разрешением меньше теплового колебания моле- 
кул [8-10]. Для возбуждения динамического процесса в материале используется источник накачки („рump source" $)$, а само исследование происходит под действием сверхкороткого электронного пучка (,probe source“) на основе анализа дифракционной картины, регистрируемой детектором, после пролета образца частицами. Условием возникновения данной картины является критерий Брэгга [11]

$$
n \lambda=2 d \sin \theta,
$$

где $n=1,2,3 \ldots-$ порядок дифракции, $\lambda-$ волна Де Бройля для электрона, $d-$ период кристаллической решетки, $\theta-$ угол Брэгга. При его выполнении электроны будут рассеиваться на узлах кристаллической решетки на угол $2 \theta$, образуя дифракционную картину на детектирующей плоскости. По свойствам данной картины можно судить о строении кристаллической решетки материала, о ее свойствах и процессах, происходящих в ней.

Длительность электронного пучка для системы быстрой дифрактоскопии определяется временным разрешением исследуемых процессов. Как правило, это $<1$ ps [8-10]. Энергия пучка должна обеспечить необходимую длину волны Де Бройля для выполнения условия (1). Количество электронов в пучке необходимо иметь достаточное для регистрации CCD-камерой, при этом заряд не должен быть большим, чтобы не увеличивать кулоновские силы.

Угловое расхождение частиц в пучке для электронной дифрактоскопии можно оценить следующим образом. На рис. 1 показана картина прохождения электронов через кристаллическую решетку материала. Рассеянные на узлах кристаллической решетки частицы отклоняются на двойной угол Брэгга $2 \theta$ и попадают в точку с радиусом $R$. Частицы, у которых имеется угловой разброс $\pm \alpha$, также отклоняются на угол $2 \theta$, но прилетают уже в положение с радиусами $R_{1,2}$. Если считать, что углы $2 \theta$ и $\alpha$ малы, то $R \approx 2 L \theta$, а $R_{1} \approx L(2 \theta-\alpha)$ и $R_{1} \approx L(2 \theta+\alpha)$, где $L-$ расстояние от узла кристаллической решетки, на котором произошло рассеяние, и плоскостью дифракционной картины. В этом случае $\Delta R=R_{2}-R_{1}=2 \alpha L$. Для достаточно качественного распознавания дифракционной картины радиус кольца $R$ должен быть хотя бы на порядок больше, чем размытие ширины кольца $\Delta R$, вызванное угловым разбросом в пучке, т.е. $R / \Delta R \approx \theta / \alpha \sim 10$. Из условия Брэгга (1) при малом $\theta$ можно записать, что $\lambda \approx 2 d \theta$, тогда $R / \Delta R \approx \theta / \alpha \approx \lambda / 2 d \alpha \sim 10$, откуда $\alpha \sim \lambda / 20 d$. Длина волны ДеБройля для электронов $\lambda=h / p$, где $h-$ постоянная Планка, $p-$ импульс частиц. При энергии $4 \mathrm{MeV}$ она составит $\lambda \approx 0.3 \mathrm{pm}$. Для характерного периода кристаллической решетки $d \approx 2$ А получаем $\alpha \sim 0.1 \mathrm{mrad}$.

С учетом вышесказанного получаются следующие оптимальные параметры пучка для системы дифрактоскопии: длительность $-100-200 \mathrm{fs}$, энергия $-3-5 \mathrm{MeV}$,

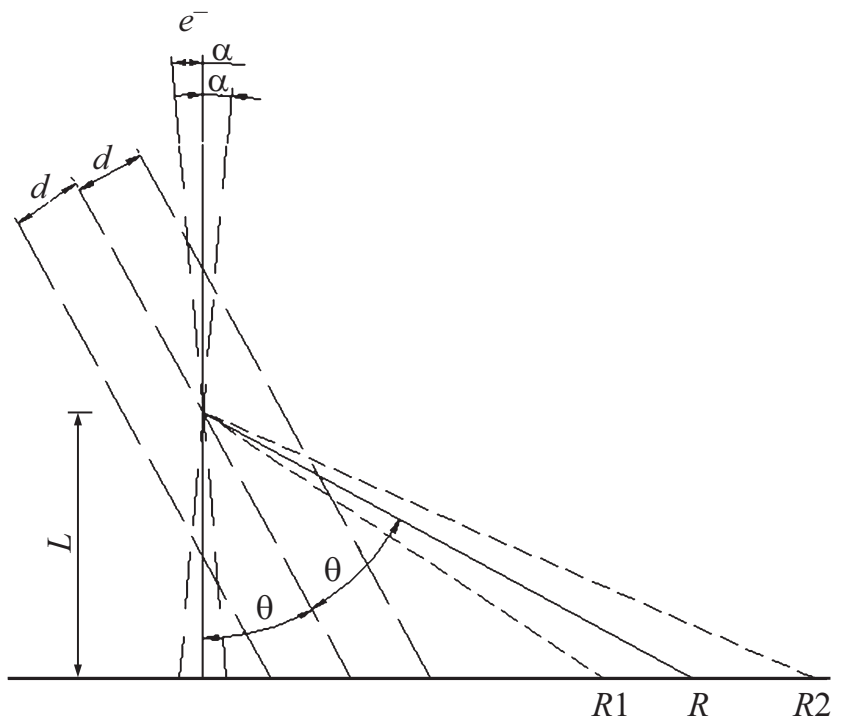

Рис. 1. Прохождение электронов через кристаллическую решетку материала.

заряд $-2-0.1 \mathrm{pC}\left(10^{7}-10^{6}\right.$ электронов в импульсе $)$, угловое расхождение $-0.1 \mathrm{mrad}$, частота повторения $\geq 10 \mathrm{~Hz}$

Получить пучок электронов с длительностью менее 1 ps можно либо с помощью соответствующего лазера, что сильно удорожит установку, либо с помощью дополнительного группирователя [12]. В этом случае требуется получение коррелированного энергетического разброса в пучке, а эффективность группировки будет сильно зависеть от линейности полученного частицами энергетического разброса. С учетом этого для ВЧ структур $S$-диапазона оптимальной начальной длиной пучка является длительность в несколько пикосекунд. В результате можно выделить требуемые параметры пучка на выходе фотопушки с учетом того, что средняя энергия пучка при группировке не меняется: длительность $\sim 2 \mathrm{ps}$, энергия $-3-5 \mathrm{MeV}$, заряд $-2-0.1 \mathrm{pC}$, угловое расхождение - $0.1 \mathrm{mrad}$.

Для плазменного кильватерного ускорения $[13,14]$ длина пучка $\sigma_{z}$, необходимого для создания кильватерной волны, определяется ленгмюровской длиной волны $[15,16]$

$$
\sigma_{z} \sim \frac{c}{\omega_{p}}
$$

где $c$ - скорость света в вакууме, $\omega_{p}=\sqrt{4 \pi n_{p} e^{2} / m}-$ плазменная длина волны, $n_{p}-$ плотность плазмы, $e$ и $m$ - заряд и масса электрона соответственно. Для создания напряженности электрического поля в несколько гигавольт на метр нужно использовать плазму с плотностью, не меньшей $n_{p} \sim 10^{15} \mathrm{~cm}^{-3}$. В этом случае, согласно (2), характерная длина пучка составит $\sigma_{z} \sim 0.2 \mathrm{~mm}\left(\sigma_{t} \sim 0.6 \mathrm{ps}\right)$. Заряд пучка, требуемый для раскачки плазменных колебаний максимальной амплитуды (определяемой нелинейным опрокидыванием волны), 
равен заряду плазменных электронов в объеме $\left(c / \omega_{p}\right)^{3}$, т.е. $q \sim e n_{p} \sigma_{z}^{3} \sim 1 \mathrm{nC}$. Величина необходимого эмиттанса пучка зависит от глубины потенциальной ямы кильватерной волны, образующейся в плазме, и от энергии частиц, поэтому он будет определяться последующим ускорителем, а не ВЧ фотопушкой.

Для исследований в области возбуждения электромагнитных полей миллиметровых длин волн в качестве возможного претендента может рассматриваться пучок из инжекционного комплекса ВЭПП-5 ИЯФ СО РАН $[17,18]$ с его последующей группировкой в сгустки с пространственным периодом несколько миллиметров. Полученные таким образом пучки способны возбуждать электромагнитные волны миллиметрового диапазона, которые могут быть использованы в ускорительной технике и физике плазмы $[19,20]$.

Для группировки высокоэнергетического пучка из ВЭПП-5 планируется использовать поворотный магнит. При его прохождении частицы двигаются по разным траекториям в зависимости от их энергии, что может привести к сокращению продольного размера пучка. Связь длины пучка с энергетическим разбросом и параметрами магнита определяется выражением [21]

$$
\Delta L=\frac{\Delta W}{W} \int \frac{D}{R} d s,
$$

где $\Delta L-$ изменение длины пучка, $W$ и $\Delta W-$ средняя энергия пучка и ее изменение соответственно, $d s-$ элемент орбиты, $R$ - радиус магнита и $D-$ его дисперсия, которая для поворотного магнита с углом $\alpha$ определяется как

$$
D=R(1-\cos (\alpha))
$$

Таким образом, с учетом (3), (4) для получения $\Delta L=1 \mathrm{~mm}$ при $\Delta W / W \approx 2 \%$ и $R=1.5 \mathrm{~m}$ нужно иметь угол поворота $\alpha \approx 34^{\circ}$. Для получения такого энергетического разброса необходимо использовать набор резонаторов, работающих в районе частоты $100 \mathrm{GHz}$ (миллиметровый диапазон длин волн). В качестве источника ВЧ мощности в этом случае планируется использовать поле излучения электронного пучка, полученного на основе ВЧ фотопушки.

Для оценки амплитуды электрического поля, наводимого пучком в резонаторе, необходимо знать параметр потерь пучка $k$, который определяется через шунтовое сопротивление $R_{s h}$, собственную добротность $Q_{0}$ и частоту $\omega_{0}$ резонатора [22] как

$$
k=\frac{\omega_{0} R_{s h}}{4 Q_{0}} .
$$

Для идеального цилиндрического резонатора, работающего на моде колебания $E_{010}$, распределение электромагнитных полей хорошо известно [23]. С учетом этого нетрудно получить выражения для запасенной энергии внутри резонатора и мощности потерь в его стенках, откуда получаются значения для шунтового сопротивления и собственной добротности. В итоге параметр потерь можно записать в виде

$$
k=\frac{L_{c} \omega_{0}^{2}}{2 \varepsilon_{0} \pi c^{2} v_{01}^{2} J_{1}^{2}\left(v_{01}\right)},
$$

где $L_{c}$ - длина резонатора, $\varepsilon_{0}$ - диэлектрическая постоянная, $v_{01}=2.405-$ первый корень функции Бесселя нулевого порядка, $J_{1}-$ функция Бесселя первого порядка. Напряжение $U_{q}$, которое возбудит пучок с зарядом $q$ в резонаторе, равно [22] $U_{q}=2 k q$. Если сгусток с зарядом $q$ не является точечным, а имеет некоторое распределение, то это можно учесть, вводя форм-фактор $F$.

Пусть заряд $q$ наводит в резонаторе с частотой $\omega_{0}$ напряжение $U_{0}$, тогда элементарный заряд $d q$ наведет в нем напряжение $d U_{q}=U_{0}(d q / q)$. Если $d q$ влетает в резонатор в момент времени $t_{0}$, то $d U_{q}=U_{0} \times$ $\times \exp \left(i \omega_{0}\left(t-t_{0}\right)\right) d q\left(t_{0}\right) / q$, где $i-$ мнимая единица. Для нормального распределения заряда с дисперсией $\sigma_{t}=c \sigma_{z}$ получается

$$
U_{q}(t)=U_{0} \cdot \exp \left(i \omega_{0} t\right) \cdot \exp \left(-\frac{\omega_{0}^{2} \sigma_{t}^{0}}{2}\right),
$$

т.е. наводимое им напряжение будет меньше в $F=$ $=\exp \left(-\omega_{0}^{2} \sigma_{t}^{0} / 2\right)$ pas:

$$
U_{q}=2 \cdot k \cdot q \cdot \exp \left(-\frac{\omega_{0}^{2} \sigma_{t}^{0}}{2}\right) .
$$

Для резонатора с частотой колебания $f_{0}=\omega_{0} / 2 \pi=$ $=100 \mathrm{GHz}$ и длиной $L_{c}=1.5 \mathrm{~mm}$ при $\sigma_{t}=2 \mathrm{ps}\left(\sigma_{z}=\right.$ $=0.6 \mathrm{~mm}$ ) наводимое напряжение в резонаторе пучком с $q=2 \mathrm{nC}$ будет в районе $U_{q} \approx 137 \mathrm{kV}$. Использования нескольких десятков резонаторов данного напряжения будет достаточно, чтобы получить необходимый энергетический разброс в пучке из ВЭПП-5.

В итоге параметры пучка после ВЧ фотопушки, необходимые для экспериментов по кильватерному ускорению и генерации электромагнитного излучения миллиметровых длин волн, должны быть следующими: длительность $\sim 0.6-2 \mathrm{ps}(0.2-0.6 \mathrm{~mm})$, энергия $\sim 5 \mathrm{MeV}$, заряд $-1-2 \mathrm{nC}$.

\section{Конструкция ВЧ фотопушки}

ВЧ фотопушка состоит из 1.5 ускоряющих ячеек с рабочей частотой $2856 \mathrm{MHz}$ и видом колебания $\pi$. На рис. 2 и 3 представлены модель данной пушки с распределением электрического поля и зависимость амплитуды ускоряющего поля на оси резонаторов от продольной координаты соответственно, рассчитанные с помощью кода CST-studio [24]. Ввод мощности в резонаторы выполнен на основе коаксиальной линии, что обусловлено необходимостью минимизировать прирост эмиттанса за счет достижения максимальной симметрии 
ВЧ поля в резонаторах. Коэффициент стоячей волны на рабочем виде колебания $\rho=1.1$. Параметры пушки составили: рабочая частота $2856 \mathrm{MHz}$, мода колебаний $\pi$, добротность резонаторов 12000 , выходная энергия пучка до $5 \mathrm{MeV}$, длина первой ячейки $2.48 \mathrm{~cm}$, длина второй ячейки $5.23 \mathrm{~cm}$, диаметр ячеек $8.2 \mathrm{~cm}$.

На рис. 4 приведены результаты моделирования в коде ASTRA [25] зависимости энергии пучка электронов на выходе фотопушки от начальной фазы инжекции для разных градиентов ускорения, полученных на основе распределения электрического поля, показанного

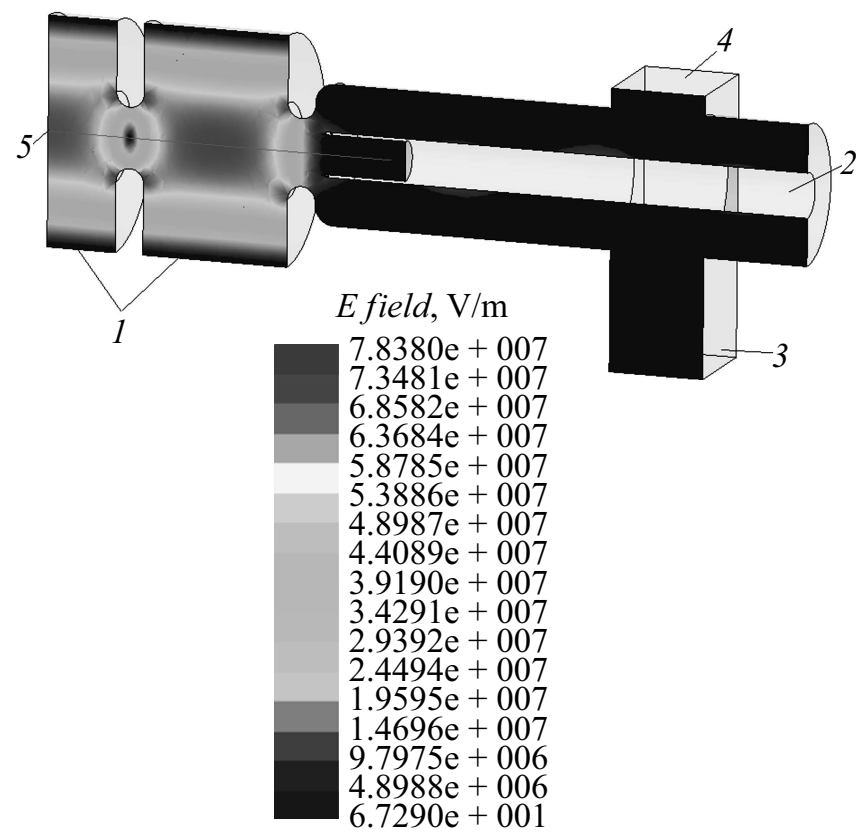

Рис. 2. Общий вид ВЧ фотопушки: 1 - ускоряющие резонаторы, 2 - канал для ввода лазера и пролета пучка, 3 - ввод ВЧ мощности, 4 - порт вакуумной откачки, 5 - положение фотокатода. Приведенное электрическое поле в ускоряющих резонаторах соответствует входной СВЧ мощности в $4 \mathrm{MW}$.

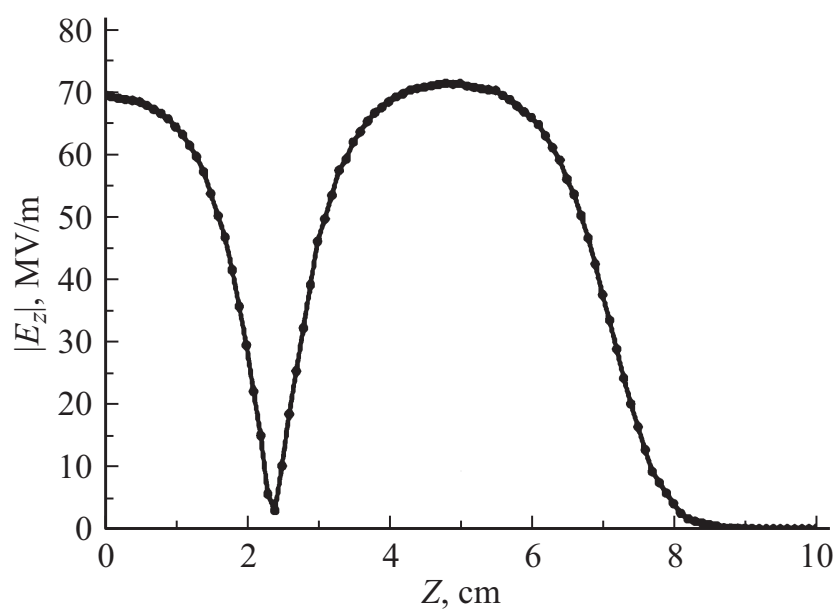

Рис. 3. Зависимость амплитуды ускоряющего поля на оси резонаторов от продольной координаты при входной мощности $4 \mathrm{MW}$.

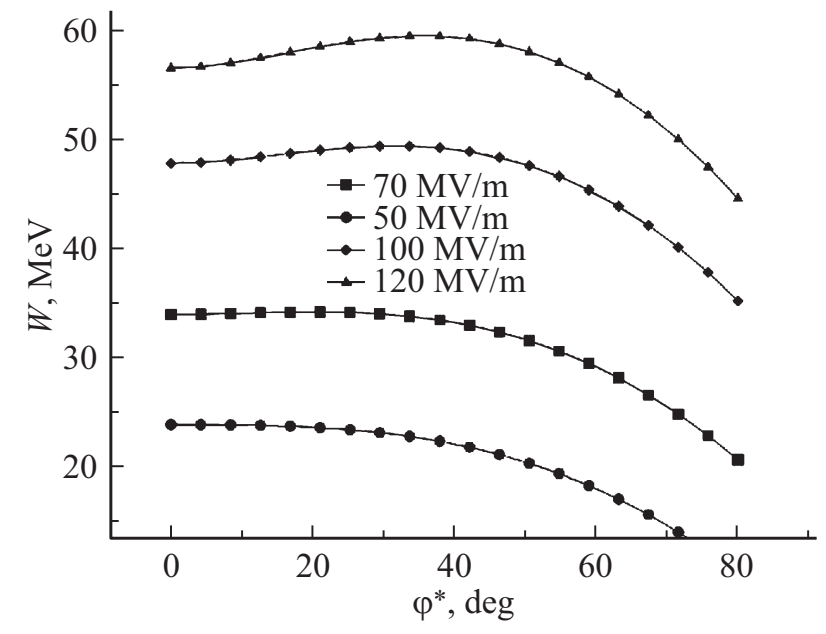

Рис. 4. Зависимость энергии электронов на выходе из пушки от начальной фазы инжекции для разных градиентов ускорения.

на рис. 3. Нулевая фаза соответствует максимальной амплитуде электрического поля. Из графиков видно, что при увеличении амплитуды ускоряющего поля начальная фаза инжекции должна увеличиваться. Это объясняется тем, что при ускорении в электрическом поле с большей напряженностью пучок быстрее становится релятивистским и не попадает в оптимальную ускоряющую фазу. Если инжектировать частицы с начальной фазой, соответствующей области поля с меньшей напряженностью, то градиент ускорения можно уменьшить, что дает возможность пучку „скользить“ по фазе переменного электрического поля и в итоге попасть в оптимальную ускоряющую фазу.

\section{Расчет динамики пучка в ВЧ фотопушке}

Для всех случаев моделирование динамики частиц осуществлялось при помощи кода ASTRA [25].

Для быстрой электронной дифрактоскопии в соответствии с выбранными оптимальными параметрами расчет проводился для двух значений зарядов пучка 0.1 и 2 рС с нормальным продольным и поперечным распределениями частиц. Значения магнитного поля соленоида для каждого заряда и начального диаметра пучка представлены в табл. 1. При этом геометрические парамет-

Таблица 1. Амплитуды магнитного фокусирующего поля

\begin{tabular}{c|c|c}
\hline Заряд пучка & $\begin{array}{c}\text { Поперечная дисперсия } \\
\text { пучка, } \mathrm{mm}\end{array}$ & $\begin{array}{c}\text { Поле соле- } \\
\text { ноида, T }\end{array}$ \\
\hline $0.1 \mathrm{pC}$ & 0.1 & 0.231 \\
$2 \mathrm{pC}$ & 0.4 & 0.226 \\
& 0.1 & 0.255 \\
& 0.4 & 0.235
\end{tabular}


Таблица 2. Амплитуды магнитного фокусирующего поля

\begin{tabular}{c|c|c}
\hline Заряд пучка & $\begin{array}{c}\text { Поперечная дисперсия } \\
\text { пучка, } \mathrm{mm}\end{array}$ & $\begin{array}{c}\text { Поле соле- } \\
\text { ноида, } \mathrm{T}\end{array}$ \\
\hline $1 \mathrm{nC}$ & 0.7 & 0.41 \\
$2 \mathrm{nC}$ & 1.2 & 0.41
\end{tabular}

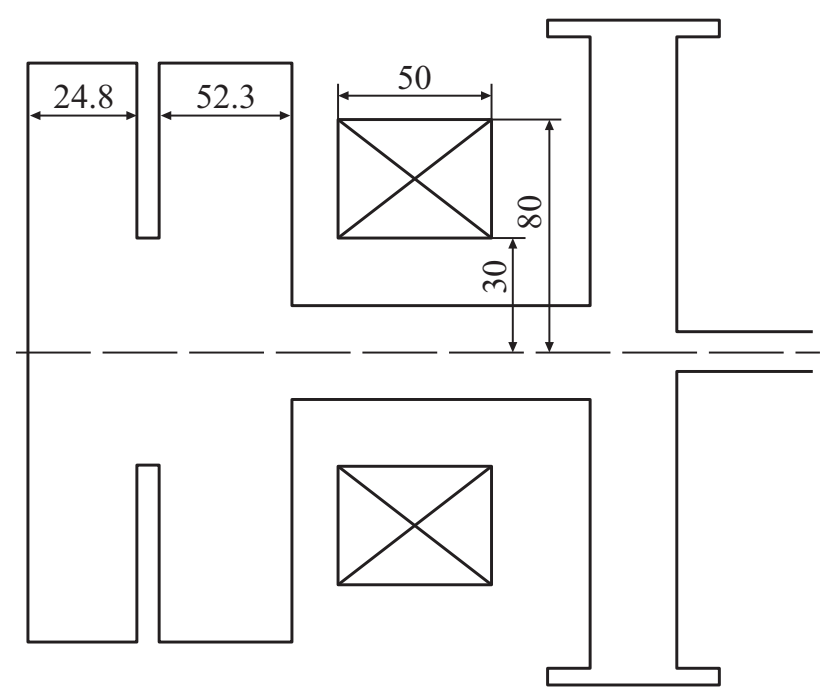

Рис. 5. Схема СВЧ пушки с соленоидом.

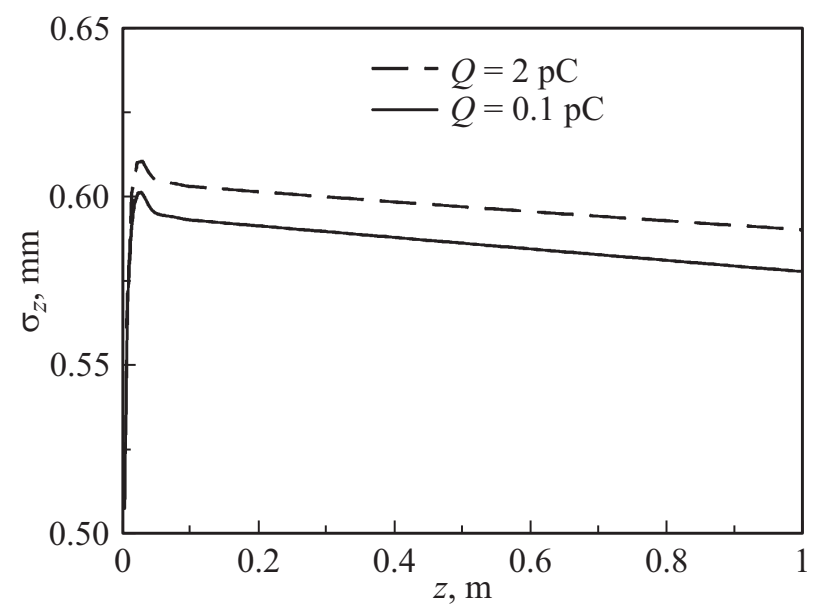

Рис. 6. Изменение продольной дисперсии пучка вдоль продольной координаты $z$.

ры соленоида были выбраны следующие: внутренний радиус $30 \mathrm{~mm}$, внешний радиус $80 \mathrm{~mm}$, длина $50 \mathrm{~mm}$. Расположение фокусирующего соленоида относительно ускоряющих ячеек показано на рис. 5 .

В результате для зарядов 0.1 и 2 рС были определены следующие начальные параметры инжекции: начальная поперечная дисперсия пучка не более $0.4 \mathrm{~mm}$, фаза инжекции $15^{\circ}$, продольная дисперсия $\sigma_{t}=5 \mathrm{ps}$. Выходная энергия составила $3.4 \mathrm{MeV}$. Изменения продольной дисперсии пучков, показанные на рис. 6, не превысили
$0.6 \mathrm{~mm}$, что соответствует $2 \mathrm{ps}$. Результаты расчетов поперечной динамики частиц приведены на рис. 7 и 8.

Из рис. 6-8 следует, что необходимые параметры пучка для экспериментов в области быстрой дифрактоскопии достигаются при использовании начальной поперечной дисперсии не более $0.4 \mathrm{~mm}$. Увеличение данного размера приводит к росту эмиттанса пучка. Амплитуды фокусирующих полей находятся в районе 0.25 Т. Из рис. 6 видно, что выходной энергии пучка достаточно для компенсации кулоновских сил расталкивания, однако для дальнейшего сокращения длины пучка необходима дополнительная группирующая структура.

На рис. 9, 10 представлено моделирование динамики пучка для двух значений зарядов 1 и 2 nC с нормальным продольным и поперечным распределениями частиц, необходимых для экспериментов в области плазменного кильватерного ускорения и генерации электромагнитных полей миллиметровых длин волн. В результате были выбраны следующие начальные параметры: фаза инжекции $10^{\circ}$, поперечная дисперсия пучка не более $1.2 \mathrm{~mm}$, продольная дисперсия пучка $\sigma_{t}=3 \mathrm{ps}$. Конечная энергия частиц составила $5.7 \mathrm{MeV}$. Амплитуда магнитного поля для каждого случая приведена в табл. 2. При расчетах использовалась схема с соленоидом, показанная на рис. 5.

Из рис. 9, 10 следует, что для работы с большими зарядами пучка необходимо увеличивать амплитуду ускоряющего поля резонаторов ВЧ фотопушки. В данном случае амплитуда была в районе $120 \mathrm{MV} / \mathrm{m}$, что требует мощности генератора около $12 \mathrm{MW}$. Кроме этого, для уменьшения начальной плотности заряда пучка его размер также приходится увеличивать. В результате полученная длина пучка составила около $\sigma_{z} \approx 0.7 \mathrm{~mm}$ $\left(\sigma_{t} \approx 2.3 \mathrm{ps}\right)$. Из рис. 9 видно, что набранной пучком энергии недостаточно для компенсации продольного расхождения частиц за счет кулоновских сил расталкивания. В этом случае необходимо использовать дополнительную ускоряющую секцию, которая позволит увеличить энергию пучка и компенсировать продольное его расхождение с возможностью последующего сокращения до требуемых размеров, указанных выше в разделе „Расчет параметров пучка ВЧ фотопушки“. Амплитуда поля соленоида в данном случае составляет примерно $0.41 \mathrm{~T}$, что является существенной величиной и может потребовать импульсного питания.

Нормализованный эмиттанс пучка для зарядов в районе $1-2 \mathrm{nC}$ в первую очередь интересен с точки зрения дальнейшей фокусировки его в апертуру резонаторов миллиметрового диапазона длин волн. Поскольку характерный размер таких резонаторов $\sim 1 \mathrm{~mm}$, апертура пролетного канала должна быть меньше - в районе $0.4 \mathrm{~mm}$. Из рис. 10 (1 и 2 ) видно, что достигаемый нормализованный эмиттанс при больших зарядах пучка составляет $\varepsilon_{n} \approx 10 \pi \cdot \mathrm{mm} \cdot \mathrm{mrad}$. Отношение бета-функции $\beta$ в области фокусировки пучка к релятивистскому фактору $\gamma$ определяется через огибающую пучка $r$ и его 

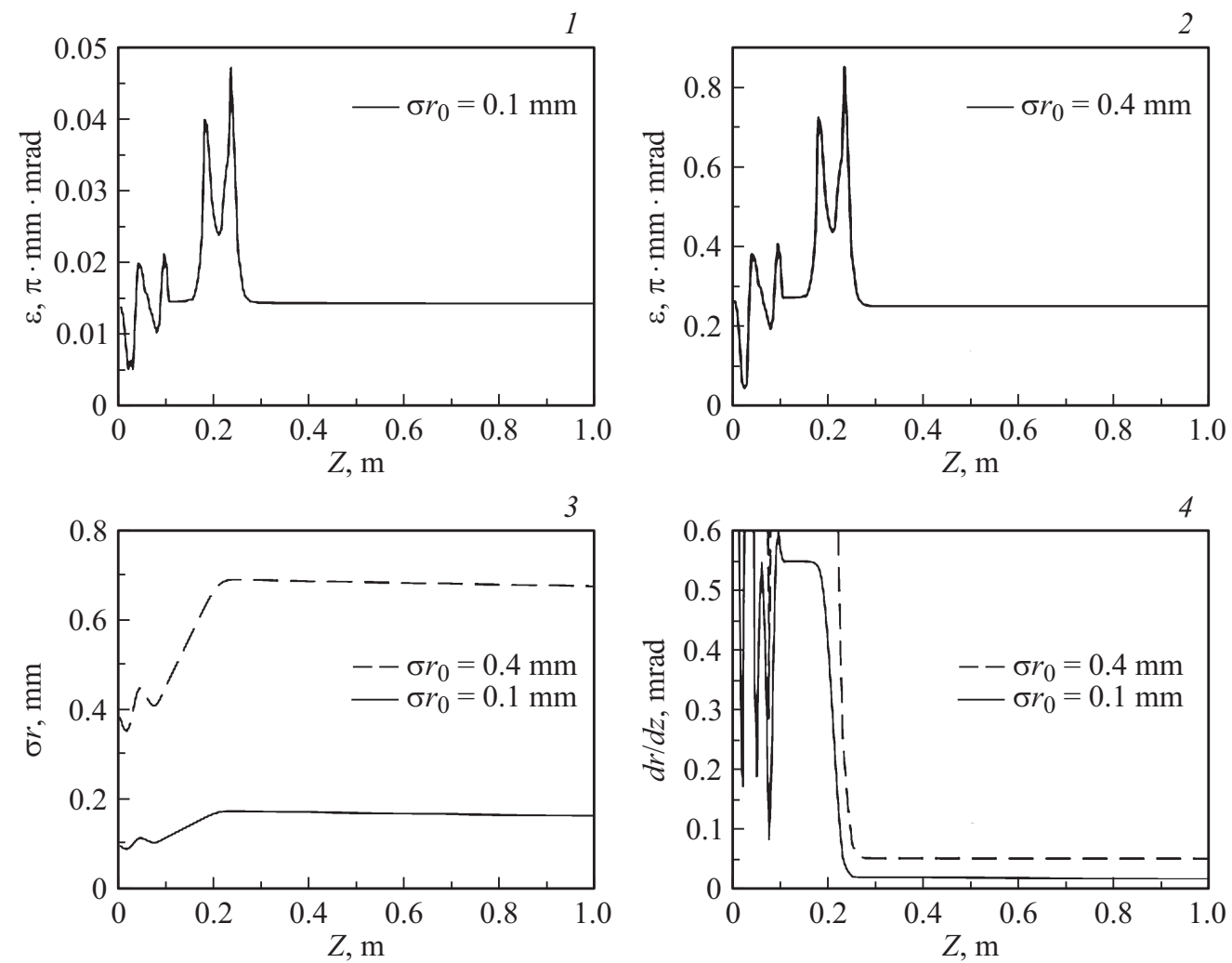

Рис. 7. Моделирование динамики частиц в фотопушке вдоль продольной координаты $z$ для заряда $0.1 \mathrm{pC}$, начальной поперечной дисперсией $\sigma_{r 0}$, фазой инжекции для всех случаев $15^{\circ}: 1-$ изменение нормализованного эмиттанса при $\sigma_{r 0}=0.1 \mathrm{~mm}, 2-$ изменение нормализованного эмиттанса при $\sigma_{r 0}=0.4 \mathrm{~mm}, 3$ - изменение поперечной дисперсии пучка, $4-$ среднеквадратичные значения углового отклонения пучка.
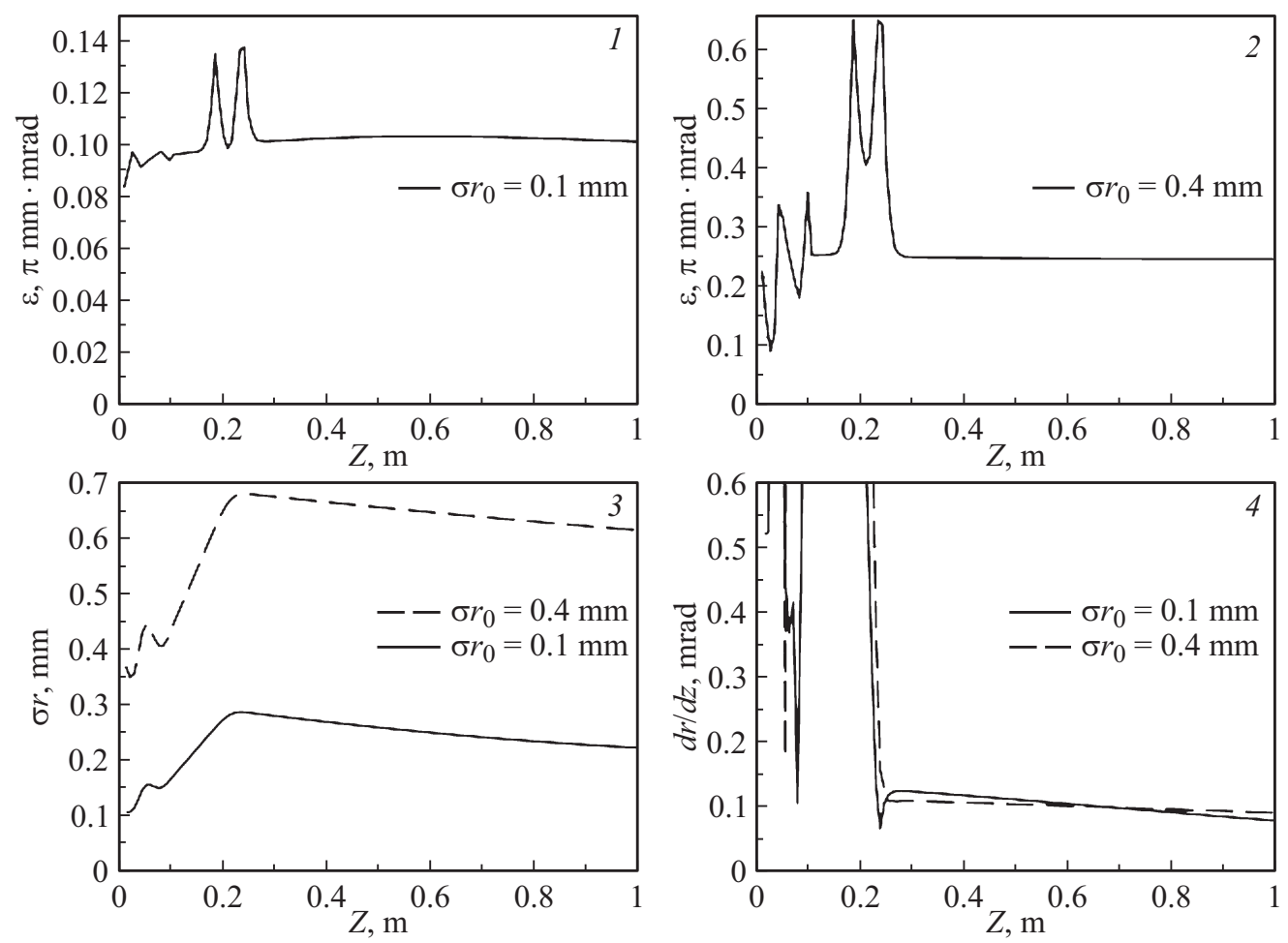

Рис. 8. Моделирование динамики частиц в фотопушке вдоль продольной координаты $z$ для заряда 2 рС, начальной поперечной дисперсией $\sigma_{r 0}$, фазой инжекции для всех случаев $15^{\circ}: 1-$ изменение нормализованного эмиттанса при $\sigma_{r 0}=0.1 \mathrm{~mm}, 2-$ изменение нормализованного эмиттанса при $\sigma_{r 0}=0.4 \mathrm{~mm}, 3$ - изменение поперечной дисперсии пучка, 4 - среднеквадратичные значения углового отклонения пучка. 
нормализованный эмиттанс [21]:

$$
\frac{\beta}{\gamma}=\frac{r^{2}}{\varepsilon_{n}}
$$

Из (8) следует, что при $r=0.2 \mathrm{~mm}$ и $\varepsilon_{n} \approx$ $\approx 10 \pi \cdot \mathrm{mm} \cdot \operatorname{mrad} \beta / \gamma=4 \cdot 10^{-3} \mathrm{~m}$. Для достижения

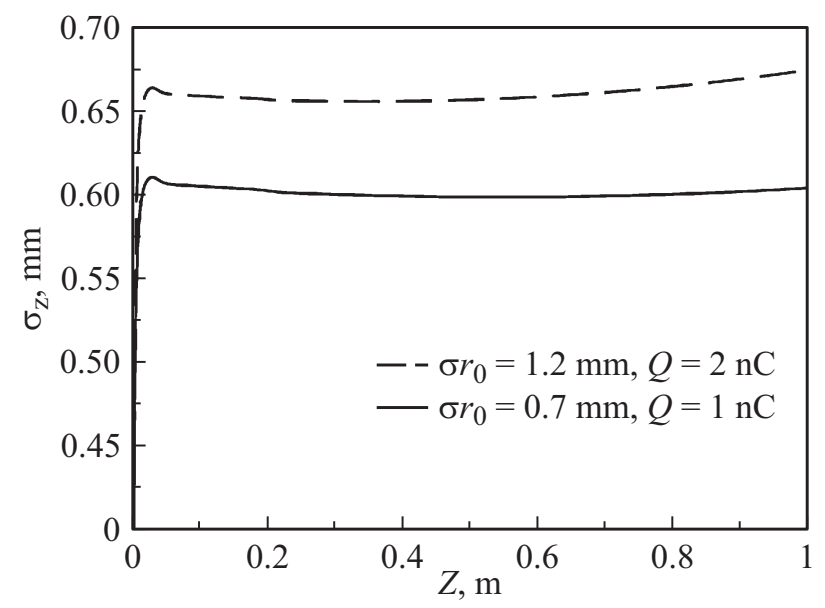

Рис. 9. Изменение продольной дисперсии пучка вдоль продольной координаты $z$ для зарядов 1 и 2 nC пучка.
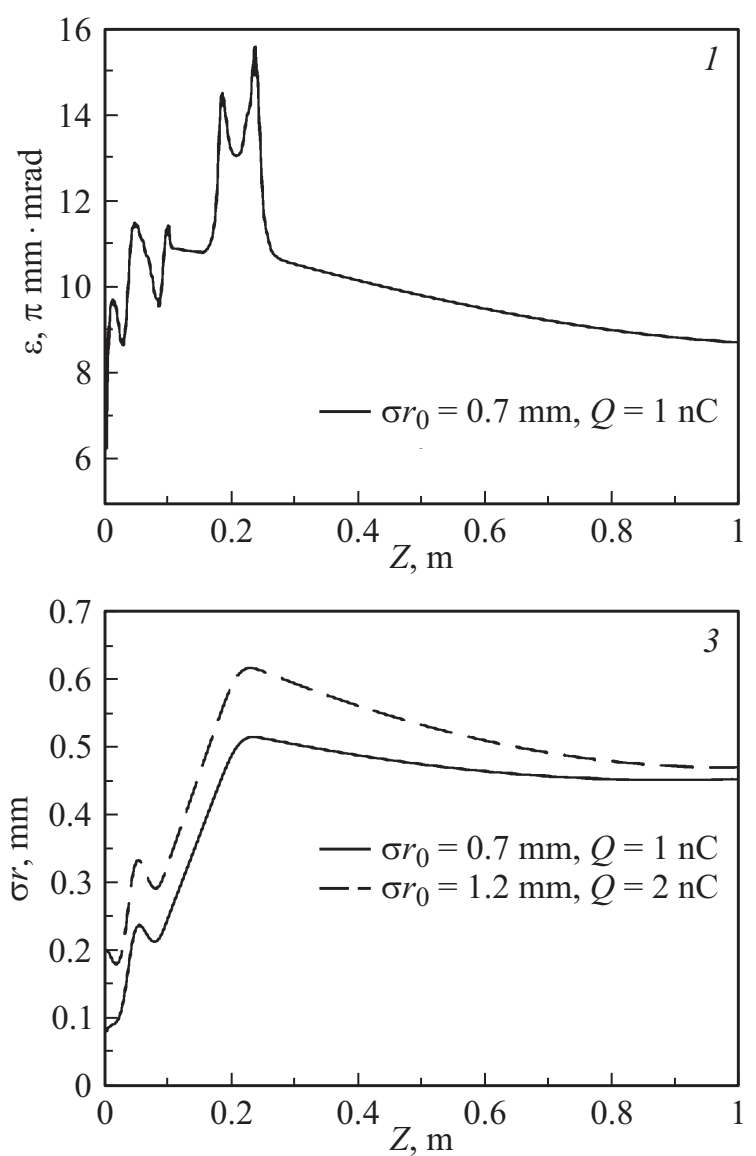

бета-функции, например в районе $0.5 \mathrm{~m}$, релятивистский фактор должен быть равен $\gamma=125$, что соответствует кинетической энергии пучка $W=62.5 \mathrm{MeV}$. Такую энергию должна обеспечить дополнительная ускоряющая структура.

\section{Заключение}

Расчет и моделирование высокочастотной фотопушки и динамики частиц показали, что не все необходимые для будущих приложений параметры пучка являются полностью достижимыми. Для всех случаев нужно использовать дополнительные системы группировки пучка. Если для малых зарядов набранной энергии в ВЧ фотопушке достаточно для компенсации кулоновских сил, то для больших зарядов необходимо дальнейшее ускорение частиц как для компенсации сил продольного расталкивания на выходе фотопушки, так и для дальнейшей фокусировки пучка. В связи с этим для перечисленных случаев последующие системы группировки будут существенно отличаться.

Для малых зарядов возможно использование группирующей системы на основе резонаторов, аналогичных используемым в ВЧ фотопушке. При этом выбор количества резонаторов и амплитуды электрического поля
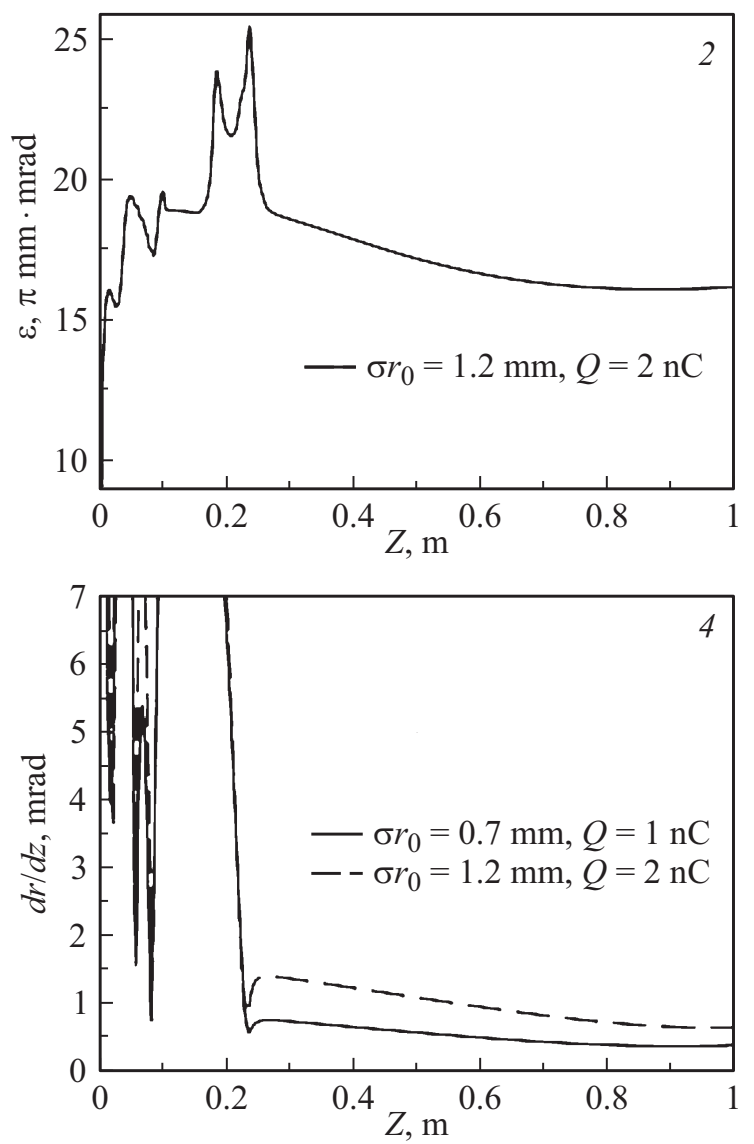

Рис. 10. Моделирование динамики частиц в фотопушке вдоль продольной координаты $z$ для зарядов 1 и $2 \mathrm{nC:} 1-$ изменение нормализованного эмиттанса при $\sigma_{r 0}=0.7 \mathrm{~mm}$ и зарядом $1 \mathrm{nC}, 2-$ изменение нормализованного эмиттанса при $\sigma_{r 0}=1.2 \mathrm{~mm}$ и зарядом $2 \mathrm{nC}, 3$ - изменение поперечной дисперсии пучка, 4 - среднеквадратичные значения углового отклонения пучка. 
зависит от последующего дрейфового промежутка. Для больших зарядов с учетом необходимого последующего ускорения пучка более целесообразным, возможно, является использование структуры с бегущим типом волны. Здесь задача значительно сложнее задачи с малым зарядом пучка и требуется отдельная исследовательская работа, которая позволит выбрать оптимальный тип группирователя.

В настоящее время сконструирован прототип ВЧ фотопушки, который позволит измерить электродинамические характеристики устройства и проверить его работу при низком вакууме и высокой СВЧ мощности. Выбор частоты генератора $2856 \mathrm{MHz}$ позволяет использовать некоторые элементы линейного ускорителя инжекционного комплекса ВЭПП-5 ИЯФ СО РАН и обеспечит дальнейшую синхронизацию работы фотопушки и инжекционного комплекса.

Работа поддержана Российским научным фондом (№ 14-50-00080).

\section{Список литературы}

[1] Assmann R., Behrens C., Brinkmann R. et al. // Proc. of IPAC. 2014. P. $1466-1469$.

[2] Mc Intosh P.A., Angal-Kalinin D., Bliss N. et al. // Proc. of IPAC. 2014. P. 2471-2474.

[3] Vardanyan T., Amatuni G., Avagyan V. et al. // Proc. of FEL. 2014. P. 561-563.

[4] Электронный ресурс. Режим доступа: https://www.rp-photonics.com/femtosecond_lasers.html

[5] Kwang-Je KIM. RF and space-charge effect in laser-driven RF electron guns. CA 94720, USA.

[6] Dowell D.H., Schmerge J.F. The quantum efficiency and thermal emittance of metal photocathodes, SLAC, Menlo Park, CA, USA,

[7] Teichert J., Xiang R., Verschuur W.J. Report on photocathodes, CARE Report-05-028-PHIN.

[8] Электронный ресурс. Режим доступа: http://pbpl.physics.ucla.edu/UESDM_2012/Talks/Pengfei\%20Zhu\%20UE SDM_2012.pdf.

[9] Williams D.B., Carter C.B. Transmission electron microscopy. Springer, 2nd edition. 2009.

[10] Xiang D., Fu F., Zhang $J$. et al. Accelerator-based Single-shot Ultrafast Transmission Electron Microscope with Picosecond Temporal Resolution and Nanometer Spatial Resolution. arXiv:1405.6445v1

[11] Bragg W.L. // Proc. of the Cambridge Philosophical Society. 1914. Vol. 17. N 43.

[12] Nawaz A.S., Werner H., Hoffmann M. et al. // Proc. of IPAC. 2015. P. 857-859.

[13] Костюков И.Ю., Пухов А.М. // УФН. 2015. № 85. Вып. 1. C. 89-96.

[14] Joshi C., Malka V. // New J. Physics. 2010. Vol. 12. P. 045003.

[15] Esarey E., Sprangle Ph., Krall J., Ting A. // IEEE Transaction on Plasma Science. 1996. Vol. 24. N 2.

[16] Lu W., Huang C., Zhou M.M., Mori W.B., Katsouleas T. // Phys. Plasmas. 2005. Vol. 12. P. 063101.

[17] Akimov A.V., Bak P.A., Barnyakov A.M. et al. // Proc. of IPAC. 2014. P. $538-540$
[18] Vereshchagin A.K., Vorob'ev N.S., Gornostaev P.B. et al. // Quant. Electron. 2016. Vol. 46. N 2. P. 185-188.

[19] Wang D., Antipov S., Jing C. et al. // Phys. Rev. Lett. 2016. Vol. 116. P. 054801. doi: 10.1103/PhysRevLett.116.054801

[20] Logatchov P., Lotov K., Petrenko A. // Nuclear Instr. 2006. Vol. 558. N 1. P. 314-316.

[21] General accelerator physics. CERN accelerator school. Ed. Bryan P., Turner S. 1985. Vol. 1. P. 47.

[22] Wangler T. Principles of RF Linear Accelerators. Wiley-VCH Verlag GmbH\&Co, 2008. P. 341-344.

[23] Wangler T. Principles of RF Linear Accelerators. Wiley-VCH Verlag GmbH\&Co, 2008. P. 26.

[24] Электронный ресурс. Режим доступа: https://www.cst.com

[25] Floettmann $K$. Astra User`s Manual, http://www.desy.de mpyflo/Astra_docomentation 